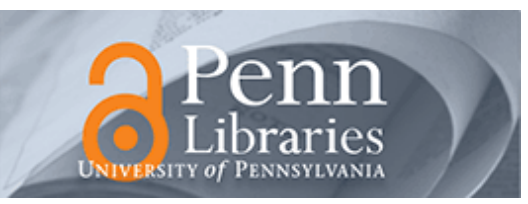

University of Pennsylvania ScholarlyCommons

January 2008

\title{
Experimental Verification of Epsilon-Near-Zero Metamaterial Coupling and Energy Squeezing Using a Microwave Waveguide
}

Brian Edwards

University of Pennsylvania

Andrea Alù

University of Pennsylvania

Michael E. Young

University of Pennsylvania

Mário Silveirinha

Universidade de Coimbra

Nader Engheta

University of Pennsylvania, engheta@seas.upenn.edu

Follow this and additional works at: https://repository.upenn.edu/ese_papers

\section{Recommended Citation}

Brian Edwards, Andrea Alù, Michael E. Young, Mário Silveirinha, and Nader Engheta, "Experimental Verification of Epsilon-Near-Zero Metamaterial Coupling and Energy Squeezing Using a Microwave Waveguide", . January 2008.

Copyright American Physical Society. Published in Physical Review Letters, Volume 100, Issue 3, Article 033903, January 2008, 4 pages.

Publisher URL: http://dx.doi.org/10.1103/PhysRevLett.100.033903

This paper is posted at ScholarlyCommons. https://repository.upenn.edu/ese_papers/332

For more information, please contact repository@pobox.upenn.edu. 


\title{
Experimental Verification of Epsilon-Near-Zero Metamaterial Coupling and Energy Squeezing Using a Microwave Waveguide
}

\author{
Abstract \\ Utilizing a microwave setup, we experimentally verify our recently developed theory of energy squeezing \\ and tunneling [Phys. Rev. Lett. 97, 157403 (2006)] through an ultranarrow waveguide channel that mimics \\ zero-permittivity properties. Exploiting the infinite phase velocity supported by a waveguide transition \\ section at cutoff, we test our theory of tunneling in this zero-permittivity region without use of resonant \\ inclusions. This "supercoupling" is shown to have unique anomalous properties: an almost uniform phase \\ along the narrow channel and weak dependence over its geometry.

\section{Keywords} \\ electromagnetic wave propagation, radiowave propagation, optical waveguides, couplers, and arrays \\ (integrated optics), electromagnetic (nonlaser) radiation interactions with plasma, plasma interactions \\ with antennas, plasma-filled waveguides

\section{Comments} \\ Copyright American Physical Society. Published in Physical Review Letters, Volume 100, Issue 3, Article \\ 033903, January 2008, 4 pages. \\ Publisher URL: http://dx.doi.org/10.1103/PhysRevLett.100.033903
}




\title{
Experimental Verification of Epsilon-Near-Zero Metamaterial Coupling and Energy Squeezing Using a Microwave Waveguide
}

\author{
Brian Edwards, ${ }^{1}$ Andrea Alù, ${ }^{1}$ Michael E. Young, ${ }^{1}$ Mário Silveirinha, ${ }^{1,2}$ and Nader Engheta ${ }^{1, *}$ \\ ${ }^{1}$ Department of Electrical and Systems Engineering, University of Pennsylvania, Philadelphia, Pennsylvania 19104 USA \\ ${ }^{2}$ Department of Electrical Engineering, Instituto de Telecomunicações, Universidade de Coimbra, 3030 Coimbra, Portugal
} (Received 14 November 2007; revised manuscript received 8 January 2008; published 25 January 2008)

Utilizing a microwave setup, we experimentally verify our recently developed theory of energy squeezing and tunneling [Phys. Rev. Lett. 97, 157403 (2006)] through an ultranarrow waveguide channel that mimics zero-permittivity properties. Exploiting the infinite phase velocity supported by a waveguide transition section at cutoff, we test our theory of tunneling in this zero-permittivity region without use of resonant inclusions. This "supercoupling" is shown to have unique anomalous properties: an almost uniform phase along the narrow channel and weak dependence over its geometry.

DOI: 10.1103/PhysRevLett.100.033903

PACS numbers: 41.20.Jb, 42.82.Et, 52.40.Db, 52.40.Fd

Metamaterials with specifically tailored values of permittivity $\varepsilon$ and/or permeability $\mu$ have recently drawn considerable attention in the scientific community due to their exotic electromagnetic properties and potential breakthroughs in various fields. Although a major effort is being invested into constructing metamaterials with simultaneously negative $\varepsilon$ and $\mu$ for their potential applications at microwaves and optics (e.g., superlensing [1]), metamaterials with $\varepsilon$-near-zero (ENZ) have also emerged into the focus of extensive research for their anomalous features at microwave and optical frequencies [2-9]. In particular, the inherent negative polarizability and fast-wave propagation in ENZ metamaterials allow the envisioning of potential applications in transparency and cloaking devices [2-4] and pattern reshaping [5-7].

As another exciting application for ENZ materials, the possibility of "squeezing" a major portion of the impinging guided electromagnetic energy into an ultranarrow channel filled with ENZ metamaterials has been theoretically envisioned in recent works [8,9]. This phenomenon, associated with the effectively "infinite" phase velocity of the wave propagation in an ENZ material, exhibits some anomalous features different from conventional resonant tunneling through a waveguide junction below cutoff $[10,11]$ : it is fundamentally independent of the length of the narrow junction and, more generally, of its overall geometry, provided that the total longitudinal crosssectional area of the ENZ channel remains sufficiently small $[8,9]$. This is, of course, counterintuitive, as one would expect an abrupt occlusion of a waveguide (i.e., a sharp variation of a waveguide's transverse cross section) to lead to large reflections. However, this supercoupling occurs even in the limit in which the channel's longitudinal cross-sectional area goes to zero. Since the electromagnetic wave does not really "propagate" in an ENZ medium, but rather "tunnels" through it with an effectively infinite phase velocity (zero-phase delay), the transmission properties of the channel are simply determined by its entrance and exit faces [8,9]. In the following, using a microwave waveguide setup we experimentally test and verify the properties of this counterintuitive supercoupling phenomenon.

The common and established technique to realize metamaterials with desired effective electromagnetic properties relies on the use of relatively complex resonant inclusions. Depending on a given application, their use may often limit the robustness of the engineered metamaterials, due to the presence of dispersion and losses associated with the inclusions' finite size and inherent resonant properties. However, it is known that a simple, inexpensive, and robust way of realizing certain effective electromagnetic responses, by all means analogous to those of metamaterials, e.g., negative permittivity, is provided by the inherent dispersion properties of waveguides [12]. A close analogy has indeed been drawn between the modal propagation within a waveguide and plane-wave propagation in an equivalent bulk material with effective constitutive parameters, which may provide the possibility of achieving and testing some of the exotic properties of metamaterials in relevant geometries. Consider, for example, the fundamental transverse-electric $\mathrm{TE}_{10}$ mode supported by a rectangular metallic waveguide, with propagation constant $\beta=\sqrt{(2 \pi n f / c)^{2}-(\pi / w)^{2}}$, where $w$ is the waveguide $H$-plane width, $n$ is the relative refractive index of the dielectric filling the waveguide, $c$ is the speed of light in vacuum, and $f$ is the operating frequency. One may generally define an effective relative permittivity for the $\mathrm{TE}_{10}$ guided mode in a rectangular waveguide as [12]

$$
\varepsilon_{\text {eff }} / \varepsilon_{0}=n^{2}-c^{2} /\left(4 f^{2} w^{2}\right),
$$

which is inherently frequency dispersive. The effective permeability $\mu_{\text {eff }}$ for this mode remains $\mu_{0}$, independent of the frequency of operation. In other words, the dispersion of propagation inside a rectangular waveguide, when limited to this dominant mode, is known to be equivalent to that of propagation in a metamaterial with permittivity $\varepsilon_{\text {eff }}$. 
This scenario was suggested in [12] in order to effectively realize plasmalike parallel-plate artificial materials at microwave frequencies, one of the early practical realizations of a "metamaterial." More recently, effective double negative metamaterials have been realized by placing split-ring resonators (for obtaining a negative permeability) inside rectangular waveguides below cutoff [that provide a negative effective permittivity, following (1)] [13-15]. Moreover, effectively epsilon-negative metamaterials have been designed using parallel-plate waveguides in planar and cylindrical geometries for cloaking applications [2]. It is underlined here that, strictly speaking, the waveguide by itself cannot be considered a continuous "material" described by a dielectric function. However, it effectively emulates the frequency response of a plasmonic material in problems where the wave propagation is effectively two dimensional and confined to the $E$ plane [9].

Exploiting this analogy for testing our theory of supercoupling phenomenon may lead us to a remarkably simple design for an effectively ENZ channel: a hollow rectangular waveguide around the cutoff frequency of its $\mathrm{TE}_{10}$ mode may behave in various aspects as a metamaterial with $\varepsilon_{\text {eff }} \simeq 0$. Indeed, at the cutoff frequency, $\beta=0$; i.e., the phase velocity is infinite, and, thus, in a certain sense the propagation in the waveguide may be regarded as equivalent to a material with effective zero permittivity, as first suggested in [12]. This may allow, as we show in the following, an experimental verification of supercoupling and the energy squeezing phenomenon [8,9] through a deeply subwavelength, effectively ENZ channel (as narrow as $\lambda_{0} / 100$, where $\lambda_{0}$ is the free space wavelength) connecting two larger waveguide sections at microwave frequencies.

The experimental setup consists of three distinct regions in a rectangular waveguide with width $w=102 \mathrm{~mm}$, as depicted schematically in Fig. 1. The walls of this waveguide are coated with conducting metal. The regions to the left of $\mathrm{RP}_{A}$ and to the right of $\mathrm{RP}_{B}$ have height $h=56 \mathrm{~mm}$ and are filled with Teflon $(n=1.41)$, supporting a propagating $\mathrm{TE}_{10}$ mode above $f=1.04 \mathrm{GHz}$. The region between reference planes $\mathrm{RP}_{A}$ and $\mathrm{RP}_{B}$, however, consists of a very narrow channel of height $a_{\mathrm{ch}} \ll h$ filled with air $(n=1.0)$, for which the cutoff frequency is $f_{0}=$ $1.47 \mathrm{GHz}$. As discussed above, around the frequency $f_{0}$ in this central region the effective permittivity "experienced" by the impinging $\mathrm{TE}_{10}$ mode is $\varepsilon_{\text {eff }} \simeq 0$, whereas in the sections filled with Teflon the effective permittivity is $\varepsilon_{\text {eff }}=1.0$. Following [8], we therefore expect to verify a supercoupling effect, supporting anomalously high transmission at the frequency $f_{0}$ through the channel, nearly independent of its geometry, provided that its crosssectional area (i.e., its height $a_{\mathrm{ch}}$ in this case) is electrically small. As theoretically suggested in [8], tiny vertical channels $\left(L_{1}=L_{2}=a_{\mathrm{ch}}\right)$ filled with air in front and in back of the channel are considered, in order to allow the wave to penetrate into the channel region and to provide proper impedance matching. This gives the $E$-plane cross section of the "ENZ" region a characteristic " $U$ " shape. Sliding metallic blocks have been used to form the $U$ channel, allowing us to easily vary its geometry (length and height). The electrical contact between the different parts has been increased by silver-based conducting grease and clamps.

In our setup (Fig. 1), one probe at reference plane $\mathrm{RP}_{1}$ has been used to excite the waveguide, and four other probes have been used to extract the reflection from and transmission through the channel in amplitude and phase relative to the reference planes $\mathrm{RP}_{A}$ and $\mathrm{RP}_{B}$. More specifically, amplitude and phase measurements at $\mathrm{RP}_{2}$ and $\mathrm{RP}_{3}$ have been used to decompose the fields in the leftmost region into the superposition of a forward and a backward $\mathrm{TE}_{10}$ mode referenced to $\mathrm{RP}_{A}$. A similar process has been performed using $\mathrm{RP}_{4}$ and $\mathrm{RP}_{5}$ to determine a forward and a backward $\mathrm{TE}_{10}$ mode referenced to $\mathrm{RP}_{B}$ in the rightmost region. These four wave amplitudes have then been used to determine the complex reflection $\Gamma$ and transmission $T$ through the $U$ channel, which is possible assuming that $\Gamma$ and $T$ each are the same for forward and backward waves due to the symmetry of the structure. $T$ is defined such that $\arg (T)=0$ indicates a zero-phase difference between $\mathrm{RP}_{A}$ and $\mathrm{RP}_{B}$. Absorbing waveguide terminations have been used on the front and the back of the setup to reduce any interfering cavity resonances that may have made this postprocessing numerically difficult. The measurement probes have been made small enough to not significantly perturb the field. All measurements have been performed with an Agilent ENA-5071C Network Analyzer.

Our postprocessed measurement results (solid lines) for the transmission through (panels a) and reflection from

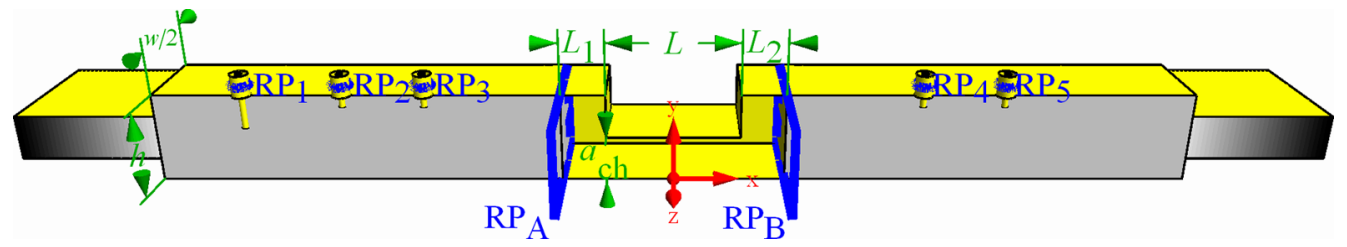

FIG. 1 (color online). Cross-sectional schematic of the experimental setup, consisting of five waveguide sections. The outermost sections are terminated with absorbing blocks (shown with gradient). Shown in gray are the copper coated Teflon waveguides supporting a $\mathrm{TE}_{10}$ mode that impinges into the air-filled $U$-shaped cutoff region at the center. The probe at $\mathrm{RP}_{1}$ is used for excitation, while the probes at $\mathrm{RP}_{2}, \mathrm{RP}_{3}, \mathrm{RP}_{4}$, and $\mathrm{RP}_{5}$ are used to extract the scattering parameters of the cutoff region referenced to the planes at $\mathrm{RP}_{A}$ and $\mathrm{RP}_{B}$. 

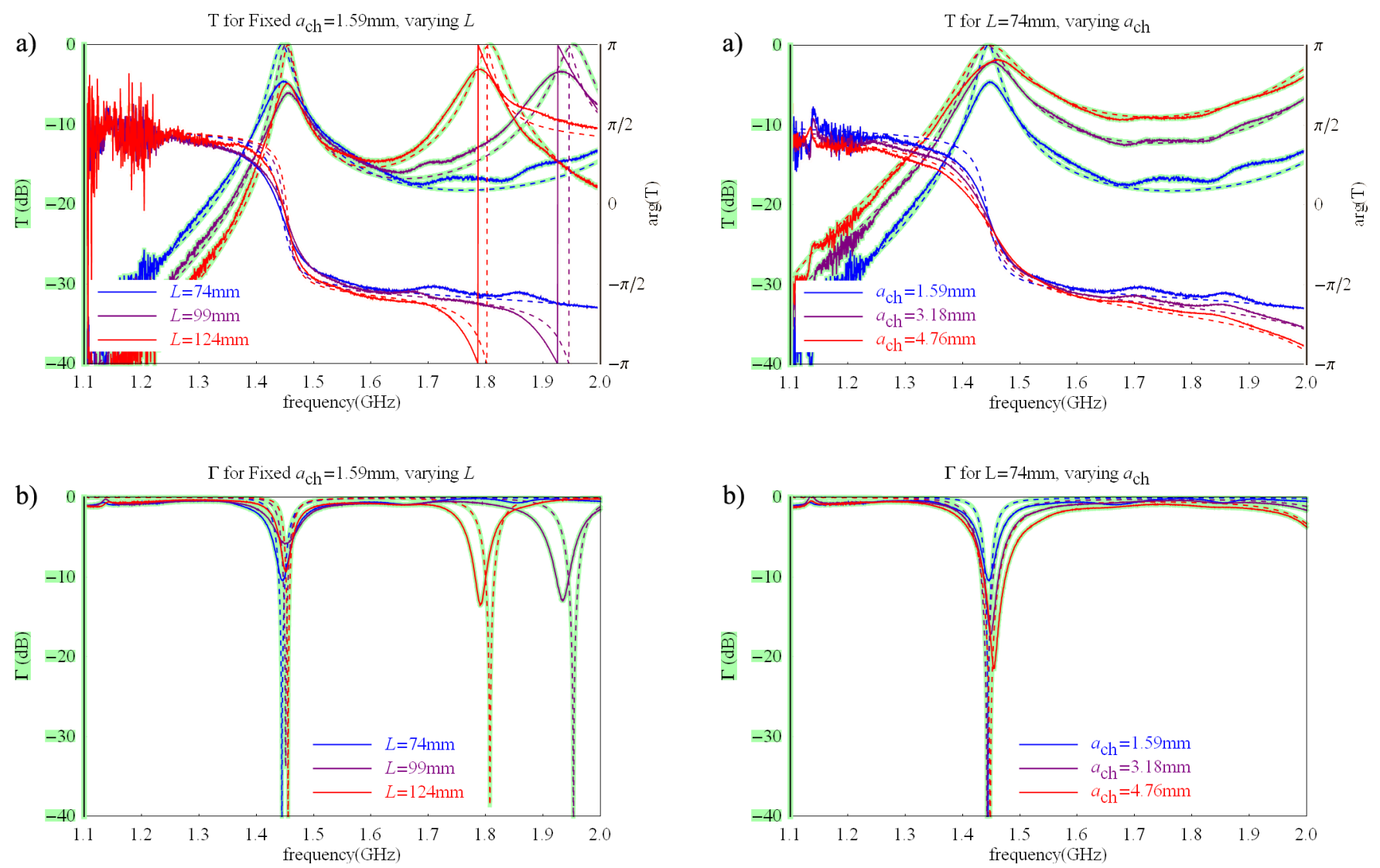

FIG. 2 (color online). Experimental (solid lines) and simulation results (dashed lines) for the transmission through (a) and reflection from (b) the $U$ channel of Fig. 1, verifying our theory of tunneling and supercoupling near the frequency $f_{0}$. Here, different channel lengths $L$ are reported for fixed $a_{\mathrm{ch}}=1.59 \mathrm{~mm}$.

(panels b) the channel are shown in Figs. 2 and 3. Simulation results (dashed lines) obtained using commercial software [16] for the same geometries are shown in the same figures. In Fig. 2 the different curves correspond to different values of the channel length for a fixed $a_{\mathrm{ch}}=$ $1.59 \mathrm{~mm}$, whereas Fig. 3 refers to different channel heights for a fixed $L=74 \mathrm{~mm}$.

Examination of the amplitudes (highlighted lines) in Figs. 2 and 3 shows that there is excellent agreement between the experimental results and the theoretical prediction for an ideal ENZ channel in a parallel-plate geometry [8]. In all these different geometries we measured peaks of resonant transmission [Figs. 2(a) and 3(a)] at the frequency $f_{0}$ to be surprisingly independent of $L$ and of $a_{\mathrm{ch}}$. The reflection plots [Figs. 2(b) and 3(b)] consistently show resonant dips at the same frequency. Given the huge geometric and impedance mismatch between the narrow channel and the two waveguide regions, the measured results demonstrate supercoupling and energy squeezing. It is also remarkable to note how a decrease in $a_{\mathrm{ch}}$ does not sensibly reduce the measured transmission peak, as seen in Fig. 3.

Additional proof of this anomalous ENZ-based supercoupling phenomenon resides in the measured phase varia-

FIG. 3 (color online). Similar to Fig. 2, but varying $a_{\mathrm{ch}}$ for fixed $L=74 \mathrm{~mm}$.

tion for the transmission across the $U$ channel (nonhighlighted lines), showing a relatively small phase difference regardless of the channel length [Fig. 2(a)] between the two reference planes. Full-wave simulations have verified that little phase and amplitude change occurs within the channel at $f_{0}$, despite being as long as $3 \lambda_{0} / 5$, consistent with propagation in an effective ENZ bulk material. This is confirmed in Fig. 4(a), where we report a time snapshot for the distribution of the normal component of the magnetic field for a channel with $L=124 \mathrm{~mm}$ and $a_{\mathrm{ch}}=4.76 \mathrm{~mm}$, as extracted from full-wave simulations.

Within the setup with sliding metallic blocks described above, we have measured transmission coefficient magnitudes of approximately $70 \%$ in terms of field amplitude for $a_{\mathrm{ch}}=h / 32$ (about $\lambda_{0} / 128$ ). While full-wave simulations predict nearly $100 \%$ transmission and zero reflection in all of the cases analyzed here, the value of the measured transmission peaks for that setup is slightly lower. This may be due to the deficient Ohmic contacts between the sliding parts forming the $U$ channel in our experimental apparatus, made more sensitive to small imperfections by the strong electric field in the tiny channel region. For similar reasons, the level of measured reflection, although minimum, is not exactly zero. (To reduce this deficiency, we have made another setup containing a $U$-transition section made of two solid metallic pieces where the inter- 


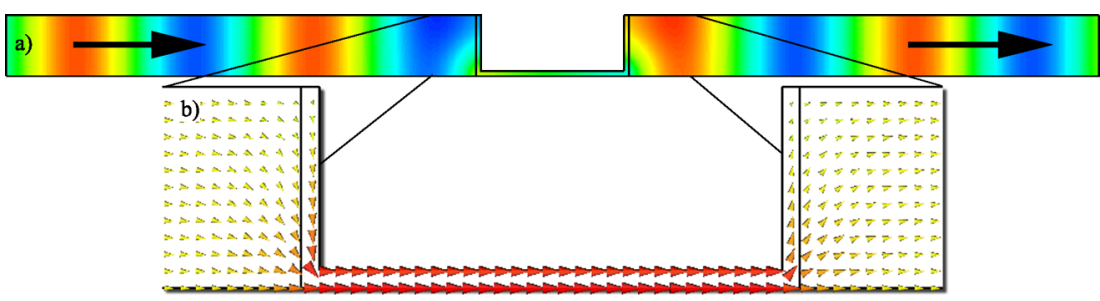

FIG. 4 (color online). (a) Simulation plot of distribution of the normal component of magnetic field (snapshot in time), showing nearly uniform phase between the two reference planes and total transmission through a channel with $L=124 \mathrm{~mm}$ and $a_{\mathrm{ch}}=$ $4.76 \mathrm{~mm}$. (b) Simulation plot of the real part of the Poynting vector distribution, showing energy flow constriction through the "effectively ENZ" region. The darker color refers to larger field amplitudes.

section lies on the $z=0$ plane, across which there are no wall currents. For this one set of values for $L=97.26 \mathrm{~mm}$ and $a_{\mathrm{ch}}=3.22 \mathrm{~mm}$, we have obtained near $100 \%$ transmission [17]. In both setups, we have been able to measure the tunneling consistent with theory and simulations, as Figs. 2 and 3 (and the figure in [17]) confirm, and therefore we have experimentally verified the supercoupling phenomenon.

This phenomenon is very distinct from the other resonant peaks visible in Fig. 2 at higher frequencies for specific values of $L$. These peaks are associated with conventional Fabry-Perot resonances with very different features compared with the phenomenon described here. Their resonant frequencies are highly dependent on the length of the channel, and their phase and amplitude distributions strongly vary across the channel at the tunneling frequency $[10,11]$. The striking effect of supercoupling is instead related to the anomalous electromagnetic properties of materials with $\varepsilon_{\text {eff }} \simeq 0$, which allows an anomalous matching with zero-phase delay in the different waveguide sections. Another important difference between this ENZrelated phenomenon and the conventional resonant tunneling below or above cutoff is its independence on the shape and geometry of the channel region. This provides the possibility of bending and rerouting the channel in any direction, without modifying its transmission properties and its tunneling frequency. Preliminary experimental results in this sense, which will be shown in a future publication, fully confirm these insights.

An interesting analogy to fluid mechanics may also be drawn: the supercoupling channel may act as a narrow "pipe" connecting two larger "conduits." In fluid mechanics, a fluid would increase in velocity within such a constriction to maintain a constant mass transported across any given cross section. Similarly, electromagnetically the energy transported across any cross section must remain constant. This is made more evident by examining the distribution of the real part of the Poynting vector in the channel region at the supercoupling frequency [Fig. 4(b)]. The tunneling is obtained by substantially increasing the power flux density in the channel, with a striking routing of energy guided in the tiny region and consequent increase of electric field in the channel region.
These experimental results support and verify the supercoupling ability of an ENZ narrow waveguide channel, which has been obtained by tailoring the modal dispersion of an empty rectangular waveguide without the need of resonant inclusions for realizing the metamaterial behavior. Waveguide geometries with segments at cutoff may in the future introduce analogous interesting devices that exploit supercoupling effects in other frequency regimes.

This work is supported in part by U.S. Office of Naval Research (ONR) Grant No. 00014 -07-1-0622.

*To whom all correspondence should be addressed. engheta@ee.upenn.edu

[1] J. B. Pendry, Phys. Rev. Lett. 85, 3966 (2000).

[2] A. Alù and N. Engheta, Phys. Rev. E 72, 016623 (2005).

[3] M. G. Silveirinha, A. Alù, and N. Engheta, Phys. Rev. E 75, 036603 (2007).

[4] A. Alù and N. Engheta, Opt. Express 15, 3318 (2007).

[5] S. Enoch, G. Tayeb, P. Sabouroux, N. Guerin, and P. A. Vincent, Phys. Rev. Lett. 89, 213902 (2002).

[6] R. W. Ziolkowski, Phys. Rev. E 70, 046608 (2004).

[7] A. Alù, M. G. Silveirinha, A. Salandrino, and N. Engheta, Phys. Rev. B 75, 155410 (2007).

[8] M. Silveirinha and N. Engheta, Phys. Rev. Lett. 97, 157403 (2006).

[9] M. G. Silveirinha and N. Engheta, Phys. Rev. B 75, 075119 (2007); 76, 245109 (2007).

[10] J. J. Hupert, IEEE Trans. Circuits Syst. 15, 279 (1968).

[11] G. Craven, Electron. Lett. 2, 251 (1966).

[12] W. Rotman, IRE Trans. Antennas Propag. 10, 82 (1962).

[13] R. Marques, J. Martel, F. Mesa, and F. Medina, Phys. Rev. Lett. 89, 183901 (2002).

[14] J. D. Baena, L. Jelinek, R. Marqués, and F. Medina, Phys. Rev. B 72, 075116 (2005).

[15] S. Hrabar, J. Bartolic, and Z. Sipus, IEEE Trans. Antennas Propag. 53, 110 (2005).

[16] CST Microwave Studio 2006B, www.cst.com.

[17] See EPAPS Document No. E-PRLTAO-100-018806 for the magnitude and the phase of the transmission coefficient for the setup containing a $U$-transition section made of solid metallic pieces. Here $L=97.26 \mathrm{~mm}$ and $a_{c h}=$ $3.22 \mathrm{~mm}$. For more information on EPAPS, see http:// www.aip.org/pubservs/epaps.html. 